the Conservancy are provided by Parliament and that public access to the reserves causes neither abuse nor friction. It is satisfactory to note that in the latest report due stress is laid upon the development of the educational, information and intelligence aspects of the Conservancy's work and the preparation of suitable publications.

\section{SICKNESS IN ENGLAND AND WALES}

A

RECENTLY published report from the Registrar General* describes three attempts to obtain statistics of morbidity, as distinct from mortality, in England and Wales. The study of mortality statistics was one of the main instruments of preventive medicine during the past hundred years. The statistics are extensive, detailed and complete. In their present form they represent the result of attempts to obtain information about human populations and not merely a by-product of administrative activity. By contrast, most existing statistics of morbidity are fragmentary and unsatisfactory, though they are of even greater potential interest than the mortality statistics. Most of the existing morbidity statistics, such as those derived from the records of Friendly Societies or hospitals, are incomplete in their coverage of the population; and most of them illustrate the limited value, for studies of general problems, of statistics originally collected for highly specialized purposes.

The three studies in the report under notice illustrate these general points, the first study being an account of the Survey of Sickness for the years 1948 and 1949. This Survey, using a designed sample, attempted to obtain information about sickness, duration of incapacity and frequency of medical consultation among the whole population. In principle, its results (here tabulated by age, sex and income of the persons interviewed) are of great importance in connexion with the operation of the National Health Service. In practice, the information obtained lacks precision, particularly as to the nature of the diseases causing incapacity. At best, the Survey provided information about what people thought was the matter with them. There is internal evidence, also, of the operation of memory effects. Nevertheless, the results of the Survey are interesting in themselves and will be even more interesting when they can be correlated with relevant statistics from other sources. Among these may be mentioned the results of an inquiry into hospital in-patient records conducted by the Registrar General, and the results of an analysis of claims to sickness benefit under the National Insurance Acts conducted by the Ministry of National Insurance. These results are apparently to be published in due course. In the meantime, it seems unfortunate that the Sickness Survey was abandoned in the interests of financial economy in 1952. Whatever the faults of this particular Survey may have been, investigations on similar lines offer the only means of obtaining morbidity statistics covering the whole population.

The second of the three studies illustrates another source of morbidity statistics, namely, records of admissions to hospital. The study is devoted to

- The Registrar General's Statistical Review of England and Wales for the year 1949: Supplement on General Morbidity, Cancer and statistics of mental disorders derived from the records of mental hospitals under the National Health Service. The results are interesting in themselves, but also serve to show the limitations of this source of information. The form designed for this inquiry proved too complicated to be satisfactorily completed by the hospitals. This is the converse of the usual situation in which the hospital administration produces records insufficiently detailed and stenderdized for the purposes of social medicine. The third study describes some of the results of the Cancer Registration Scheme relating to 1947 and 1948. Statistics for 1945 and 1946 have already been published elsewhere. This is an interim study describing an early phase of the construction of a complete statistical picture of this group of diseases. At present it covers only part of the population (that served by the hospitals taking part in the Scheme), but it achieves a high degree of diagnostic accuracy. It will in the end, no doubt, combine both accuracy and completeness. At present the tables give information about numbers of cases by age and sex and about the delay between first symptoms and starting of treatment for cancer of various sites.

\section{EARTHQUAKES DURING 1953}

$\mathrm{T}$ HE five earthquakes which did most damage during 1953 occurred on February 12 (magnitude 7) in Persia; on March 18 (magnitude 73 Turkey; during August a swarm (greatest on August 12, of magnitude 7t) near Cephalonia ; on

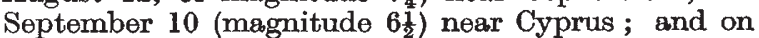
December 12 (magnitude 73 ) in Peru. In addition, nineteen other earthquakes were of magnitude 7 or more, the greatest being on November 25 (magnitude 81) near Japan.

Fourteen earthquakes had foci at depths of $200 \mathrm{~km}$. or greater, the deepest occurring on April 14 (magnitude 7) in western Brazil and on November 4 off Korea, both having a depth of focus of $650 \mathrm{~km}$.

The Persian earthquake in February occurred in the Province of Khorasan and devastated Shahrood, Torroud and neighbouring villages, killing hundreds of the inhabitants when their houses collapsed on them.

The Turkish earthquake of March 18 occurred south-east of the Dardanelles in the region of Chanak, Balikeser, Yenice and Gonen, where more than a quarter of a million pounds sterling damage was done and hundreds of people were killed by falling debris and the fires which followed the earthquake.

The swarm of earthquakes in the Greek Ionian islands of Cephalonia, Ithaca and Zante culminated in the shock of magnitude $7 \frac{1}{1}$ on August 12 and caused great damage, there being 394 deaths and a further 941 casualties (see Nature, August 22, p. 332). Argostoli, Lixouri and Zakynthos were particularly affected, and just after the earthquakes more than seventy-five thousand people were homeless. The International Seismological Association at Strasbourg reports that some 338 individual shocks occurred in the swarm of August 9-31.

The earthquake which did damage in the Paphos district of Cyprus on September 10 had a smaller magnitude than the others mentioned; but in the town of Ktima and the surrounding villages, including Stroumbi, Kithasi and Ayios Nikolaos, and even as 
far as Limassol, much damage was caused; forty people were killed, a hundred injured, and just after the earthquake some four thousand were homeless.

From the reports from sixty-one observatories throughout the world, the epicentre of the earthquake of December 12 has been calculated by $\mathbf{E}$. Peterschmitt and L. Behe at Strasbourg to be at lat. $3.7^{\circ} \mathrm{S}$., long. $80.7 \mathrm{~W}$., which is near the coast of Peru. Several people were killed as a result of this earthquake, and there was heavy property damage at Tumbes, Corrales and in the surrounding district.

Reports were received during the year directly from individual stations at Aberdeen, Arkansas, Belgrade, Cleveland (Ohio), Durham, Fayetteville, Karlsruhe, Kew, Lisbon, Lwiro (Belgian Congo), Pasadena, Perth (Western Australia), Toledo and Uccle; from collecting stations at $\mathrm{De}$ Bilt, Stuttgart, Wellington (New Zealand) and Zurich; and also from the International Seismological Association at Strasbourg and the United States Coast and Geodetic Survey in co-operation with Science Service and the Jesuit Sejsmological Association, where observations are collected and preliminary epicentres determined.

ERnest Tillotson

\section{RADIO EVIDENCE OF THE EJECTION OF VERY FAST PARTICLES FROM THE SUN}

\section{By J. P. WILD, J. A. ROBERTS and} J. D. MURRAY

Division of Radiophysics, Commonwealth Scientific and Industrial Research Organization, Australia

THE method of studying movements in the solar atmosphere by observing the spectrum of highintensity radio noise from the sun has been discussed in previous communications ${ }^{\mathbf{1}, \mathbf{2}}$. It depends on the simple hypothesis that when the spectrum shows an abrupt low-frequency cut-off, the frequency of this cut-off can be identified with the critical escapefrequency at the source, and hence used for determining the height of the source in the solar atmosphere. The hypothesis has previously been applied to the outbursts of long duration for which the characteristic slow drift of the cut-off frequency can be explained in terms of disturbances which move outwards through the solar atmosphere at velocities ${ }^{1}$ of the order of $500 \mathrm{~km}$./sec. This result was confirmed in general terms by the directional observations of Payne-Scott and Little ${ }^{3}$. More recently, the criticalfrequency hypothesis has been further substantiated by evidence that the observed cut-off must be attributed not to the natural profile of the emitted spectrum, but rather to severe attenuation of the lower frequencies in their escape from the sun ${ }^{2}$. This evidence was obtained by comparing profiles of the fundamental and the second harmonic (which is presumably unattenuated).

In the present communication we offer similar evidence for the application of the same hypothesis to another, more common, type of burst in which the frequency drift is much faster. Thence we infer the existence in the solar atmosphere of corpuscular ejections which move outwards at about one-fifth the velocity of light.

\section{Type ill Bursts}

Using the previous nomenclature ${ }^{4}$, we shall refer to the two spectral classes mentioned above as type II (slow drift, duration 5-10 min.) and type III (fast drift, duration 5-10 sec.) respectively. In the course of observations between August 1952 and August 1953 with a wide-band (40-240 Mc./s.) spectroscope, it was found that nearly all bursts, excluding those in noise storms, belonged to one of these two classes. In all, four outbursts of type II were recorded and several hundred bursts of type III. The latter

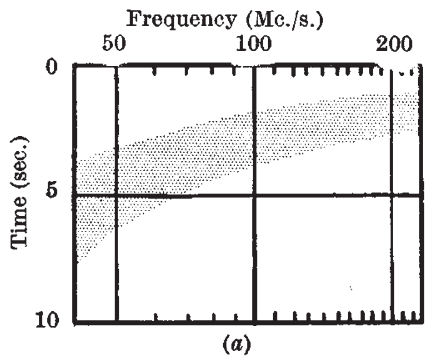

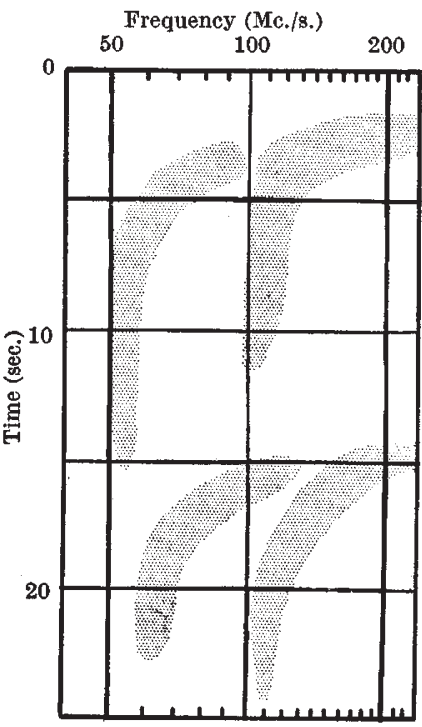

(b)

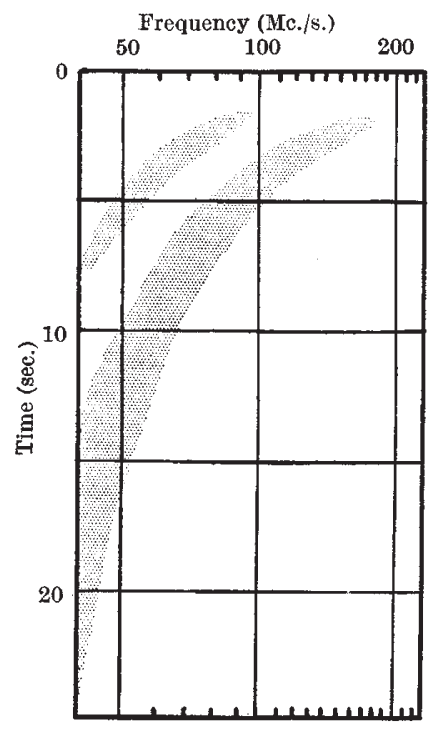

(c)

Fig. 1, Dynamic spectra of type III bursts. The shaded areas represent regions of high intensity in the frequencytime plane. (a) April 2, 1953,0447 U.T. (b) Harmonic pairs, Jan. 14, 1953, 0607 ठ.T. (c) Harmonic pair, June 5, 1953, 0135 O.T. 